\title{
Aportaciones de R. Sennett al desarrollo de la ética empresarial en el contexto del Nuevo Capitalismo
}

\author{
ALBERT MUÑOZ MIRALLES* \\ Universitat Jaume I - Castellón (España) \\ Albert.Munoz@uji.es \\ ELSA GONZÁLEZ-ESTEBAN** \\ Universitat Jaume I - Castellón (España) \\ esteban@uji.es
}

\begin{abstract}
Resumen
Este artículo tiene el objetivo de realizar una aproximación crítica al pensamiento de Richard Sennett con la mirada puesta en las aportaciones que su obra puede realizar al desarrollo de la Ética Empresarial. De ahí que el estudio busca reconocer las transformaciones que están produciéndose en el nivel organizativo, y que se traducen en la implementación de nuevas formas de producción y trabajo en el seno de las relaciones capitalistas, aspectos sumamente relevantes para ser capaces de orientar dichas relaciones en un sentido de justicia, prudencia y responsabilidad tal y como se plantea por la Ética Empresarial de corte discursivo.
\end{abstract} Palabras clave: Ética empresarial, Richard Sennett, Nuevo Capitalismo, carácter, artesanía.

\section{Contributions by R. Sennett to develop Business Ethics in the New Capitalism context}

\begin{abstract}
The objective of this article is to conduct a critical study of Richard Sennett's thinking by focusing on the contributions his work can make to develop Business Ethics. This study aims to recognise the transformations that take place in organisational terms, and are then implemented into new forms of production and work at the heart of capitalist relations, which are extremely important aspects for guiding these relations in justice, prudence and responsibility terms, as it is proposed by discourse business ethics.
\end{abstract}

Keywords: Business Ethics, Richard Sennett, New Capitalism, character, craftsman.

* Investigador Colaborador del Grupo Ética Empresarial y Filosofía Política de la Universitat Jaume I. Doctor en Ética y Democracia por la Universitat Jaume I. Recientemente ha publicado: "El potencial filosófico del concepto de carácter en Sennett" (2015). ** Profesora Titular de Filosofía Moral en la Universitat Jaume I de Castellón. Investigadora del Grupo Ética Empresarial y Filosofía Política de la Universitat Jaume I. Doctora en Ética Empresarial por la Universitat Jaume I. Es editora de Ética y Gobernanza: un cosmopolitismo para el siglo XXI (2013) y su artículo más reciente se titula "Autocontrol: A critical study on potential achievements and challenges in the search for promoting ethical advertising through advertising self-regulation system" (2017 junto a R. Feenstra). 
1. REORIENTAR ÉTICAMENTE LAS RELACIONES EMPRESARIALES EN EL CONTEXTO DE LAS ACTUALES RELACIONES CAPITALISTAS ${ }^{1}$

Desde que nació la ética empresarial o business ethics en los inicios de la década de los años 70 su pretensión sigue concentrada en tres aspectos nucleares, a saber: a) dilucidar la meta y bienes internos por las que cobra sentido y legitimidad la actividad empresarial; b) reconocer y potenciar los hábitos y valores que permiten alcanzar tales metas; y, c) diseñar los procedimientos y procesos en las organizaciones empresariales que aproximan a la toma de decisiones prudentes, justas y responsables (Cortina, et.al., 1994).

En la actualidad, especialmente tras la crisis económica vivida en el primer decenio del s. XXI, asistimos a una gran transformación de las relaciones organizativas dentro del contexto de las nuevas formas de capitalismo que han ido desarrollándose.

El estudio que aquí se plantea tiene el objetivo de realizar un análisis profundo y crítico del pensamiento de Richard Sennett, autor que ha dedicado sus esfuerzos en las últimas tres décadas a describir las nuevas formas relacionales que se establecen en el ámbito económico-organizativo, así como a proponer caminos que eviten la erosión del carácter de los individuos o su identidad en la sociedad. El acercamiento a la riqueza de la obra y propuesta de este autor se debe, como se mostrará, a que realiza una importante aportación al diagnóstico de las relaciones organizativas en el sistema capitalista que a nuestro modo de ver puede ser una importante contribución al desarrollo de la ética empresarial en nuestros días. De ahí que el estudio busca reconocer las transformaciones que están produciéndose en el nivel organizativo, y que se traducen en la implementación de nuevas formas de producción y trabajo en el seno de las relaciones capitalistas, aspectos sumamente relevantes para ser capaces de orientar dichas relaciones en un sentido de justicia, prudencia y responsabilidad.

Esta investigación está dividida en cinco partes. En primer lugar, se analiza y sitúa la descripción que Richard Sennett realiza del capitalismo en el seno de las relaciones de globalización, al que denomina Nuevo Capitalismo y que nosotros situaremos en las propuestas de la literatura especializada. En segundo lugar, se profundiza en las transformaciones que en ese contexto del Nuevo Capitalismo se están produciendo tanto en la concep-

1 Este estudio se inserta en el Proyecto de Investigación Científica y Desarrollo Tecnológico FFI2016-76753-C2-2-P, financiado por el Ministerio de Economía y Competitividad, y en las actividades del grupo de investigación de excelencia PROMETEOII/2014/082 de la Generalitat Valenciana. 
tualización del concepto de trabajo como en las estructuras de las organizaciones. En tercer lugar, se muestra de la mano de Sennett la consecuencia corrosiva que tienen tales transformaciones en el carácter de los trabajadores y sus relaciones. En cuarto lugar, se aborda el concepto de artesanía propuesto por Sennett como una vía para desplegar el carácter y evitar la corrosión del mismo. $\mathrm{Y}$, finalmente, en quinto lugar, se profundiza en qué consideraciones y orientaciones éticas se desprenderían de la utilización de este concepto de artesanía para la configuración de una ética empresarial en el contexto económico actual.

\section{LOS ENTRESIJOS DEL NUEVO CAPITALISMO: LA FLEXIBILIZACIÓN DEL TRABAJO}

Si se propone emplear, siguiendo a Sennett, la noción de Nuevo Capitalismo es debido a la importancia y alcance que están alcanzando los procesos de transformación de las estructuras institucionales y de la actividad económica en las últimas décadas, impulsados por los rápidos avances tecnológicos, por la apertura, extensión y dinamización de unos mercados globales. De lo que se trata es de realizar una aproximación crítica a la orientación que sigue la configuración actual de la economía capitalista, especialmente en la medida en que conlleva una precarización del trabajo y una merma de las oportunidades y garantías institucionales. Pues hay que cuestionarse que se trate de una prolongación inevitable de la dinámica económica inherente al capitalismo. Cabe entender, a nuestro juicio, que la fase actual representa, en buena medida, una versión actualizada de lo que en la clasificación de Albert (1991) correspondía al modelo anglosajón, oponiéndola al renano. Es preciso señalar que no se trata de una mera distinción empírica, pues se solapa con la disputa de un cariz indudablemente ideológico en el terreno de la filosofía política entre el neoliberalismo y la socialdemocracia (Crouch, 2005: 441).

El modelo anglosajón, según Albert, se basaría en la búsqueda del éxito individual en un mercado abierto y netamente competitivo, que incita a asumir riesgos con vistas a obtener un rendimiento a corto plazo. En cambio, en el modelo renano primaría la estabilidad y la cohesión social, por lo que el marco institucional en el que se desarrolla la actividad económica adquiere mayor importancia, así como se reconoce un papel más relevante al Estado como promotor de los bienes públicos. La empresa, en esta perspectiva, se concibe más bien como una comunidad de intereses entre sus distintos componentes, mientras que para el modelo rival se trataría fundamentalmente de retribuir la inversión de los accionistas (Albert, 1991). En ese sentido, la organización empresarial de tipo renano respondería a un modelo institucional de stakeholders, que revela una 
orientación bien distinta, más inclusiva y participativa, que el ofrecido por el modelo shareholder. Por ello, en el sistema renano la industria se erige como el pilar de la economía, propiciando la integración de los trabajadores en las empresas, mediante una formación en las habilidades específicas que ayudan a desarrollar una carrera en su seno (Albert \& Gonenc, 1996: 186) $)^{2}$.

El modelo renano, establecido sobre una base contractual, obtiene mayor legitimidad social, además de haberse mostrado eficiente (Albert \& Gonenc, 1996: 184). Sin embargo, la crisis del estado del bienestar propició el cuestionamiento de sus bases keynesianas y en general del modelo de un capitalismo social, imponiéndose en la práctica las tesis neoliberales. El desarrollo de la globalización conlleva una intensificación de las tendencias propias del modelo neoamericano, propiciando la hegemonía de una lógica financiera altamente especulativa, alimentada de la desregulación, las facilidades tecnológicas y técnicas financieras que prometen beneficios inmediatos. En consecuencia, la producción industrial se ve sometida a los intereses de las finanzas, provocándose un desgaste del tejido social e institucional sobre el que se elaboraba el consenso básico, al perder vigencia los valores que lo sostenían - justicia, confianza, regulación, etc.- en favor de un mayor individualismo. Pese a las dificultades del tradicional modelo renano para afrontar los retos que plantea la nueva economía global, coincidimos en la necesidad de promover sus valores intrínsecos en un escenario marcado por la incertidumbre - por ejemplo, la confianza se vuelve

2 Tras Albert, otros autores - especialmente desde el neoinstitucionalismo- han tratado de ordenar las diferentes economías empleando una formulación dicotómica similar, tomando como referencia un modelo de libre mercado típico de la economía neoclásica contrastándolo con un modelo coordinado (Crouch, 2005: 440ss.; Ebbinghaus \& Manow, 2001: 6). Así, se habla de dos modos básicos de coordinación -aunque ambos pueden darse en grados variables en cada país-: a través de mercados competitivos; o mediante la interacción estratégica de los diversos actores conforme a la teoría de juegos, lográndose el equilibrio mediante el soporte institucional. La economía de mercado no coordinada $-L M E$, dominante en los países anglófonos- se distinguiría por la prevalencia de unos mercados financieros que persiguen el beneficio a corto plazo, por una producción poco especializada, por unas relaciones y negociación laborales descentralizadas, con alta movilidad laboral y por una formación general. En cambio, en la economía coordinada - CME, propia de Alemania o Japón- prima una financiación a más largo plazo, existen conexiones más estables entre los diversos actores, se da una producción más especializada y cualificada, y ofrece una mayor permanencia y estabilidad a unos trabajadores con una formación más vocacional (Ebbinghaus \& Manow, 2001; Hall \& Gingerich, 2004). Crouch, sin embargo, critica estas clasificaciones apriorísticas por forzar una realidad empírica mucho más compleja enclaustrándola en una binarización artificiosa (Crouch, 2005: 441ss.). 
más preciada cuando las transacciones son anónimas y lejanas-, reclamándose una mayor estabilidad, justicia y legitimidad en la actividad económica y política a nivel global (Albert, 1991; Albert \& Gonenc, 1996: 188-191).

Lo importante es analizar cómo afectan estas tendencias a la organización del trabajo, para lo que la obra de Sennett ofrece ciertas claves fundamentales. Destaca la implantación de patrones más flexibles con los que las empresas de vanguardia pretenden liberarse de las rigideces impuestas por las tradicionales estructuras burocráticas piramidales y los modelos fordistas de organización del trabajo, que habían dado forma a la era del capitalismo industrial. Según la clásica definición weberiana, se trataba de una estructura nítida, integrada y bien delimitada, desarrollando un funcionamiento normalizado conforme a unas reglas definidas. La racionalización de la gestión empresarial fue impulsada gracias a la introducción de técnicas tayloristas, encontrando su realización efectiva en la producción masiva de bienes estandarizados propia del fordismo.

Sin embargo, según se está exponiendo, la diversificación de los mercados y la introducción de las nuevas tecnologías de la información, altera el funcionamiento de las organizaciones difuminando sus contornos. Ahora han de ser capaces de mantenerse en un contacto permanente y fluido con su entorno si quieren ser competitivas. Para responder a las nuevas exigencias y retos se han ido introduciendo en el mundo empresarial formas alternativas de organización, más flexibles y descentralizadas, que ofrecen respuestas más rápidas y eficientes a los cambios en el entorno.

El Nuevo Capitalismo se caracteriza así por la globalización económica, el auge de las nuevas tecnologías, la flexibilización e indeterminación de las estructuras institucionales y la pérdida de las garantías que ofrecía el estado del bienestar (Sennett, 1997: 161). Esta nueva economía asciende impulsada por el "trabajo en los sectores de la alta tecnología, las finanzas y los servicios, sostenidos por inversores internacionales y organizados en instituciones más flexibles, sensibles y centradas en el corto plazo que las rígidas jaulas burocráticas del pasado" (Sennett, 2009: 49). Su aspecto definitorio es precisamente la flexibilidad que, más allá de las facilidades para transferir capital y trabajo a través de las fronteras, se realiza a través de la nueva estructuración institucional del trabajo, desafiando la noción weberiana de una división fija de las tareas y funciones (Sennett, 2002: 45) ${ }^{3}$. No obstante, Sennett advierte que el nuevo modelo organizativo no está aún

La importancia que Sennett concede a la flexibilidad como elemento definitorio del Nuevo Capitalismo le permite designarlo, en ocasiones, como capitalismo flexible (Sennett, 2000: 9). Por ello, es insuficiente hablar sólo de globalización. 
generalizado, localizándose principalmente en sectores punteros de la economía, como la industria tecnológica o los servicios financieros. Pero lo importante es que estas empresas están actuando como una vanguardia cultural, marcando el camino a seguir al resto de organizaciones (Sennett, 1999: 3-4; 2006a: 16; 2006b: 166) ${ }^{4}$.

La transformación del modelo piramidal de organización y la crisis del sistema de trabajo fordista, propiciando la implantación de estructuras más flexibles, había sido ya percibida y analizada por otros autores. La noción de adhocracia, empleada por Toffler o Mintzberg, o la de red, desarrollada por Castells, así como la etiqueta de post-fordismo para designar los cambios en la organización del trabajo, ayudan a comprender la dirección y el alcance de las transformaciones organizativas. Pero también podría recurrirse complementariamente a los estudios realizados por Boltanski y Chiapello (2002) o por Fleming (2015) sobre las consecuencias que tal flexibilidad de las relaciones laborales, así como las estructuras reticulares, tienen sobre el trabajo y los mecanismos que cabría activar para frenar sus efectos perversos y destructores.

Ya en los años 80, Toffler percibió que la producción estandarizada dejaba de ser eficiente ante una sociedad cambiante y diversa, requiriéndose la introducción de nuevos modelos organizativos, más dinámicos y menos jerarquizados - adhocráticos - para impulsar la flexibilización (Toffler, 1995: 107-108 y 127-132). Mintzberg profundizó en el concepto de adhocracia, tratándose de un modelo de organización innovador, flexible, que rehúye los procedimientos fijos, pues sus funciones y composición varían constantemente, adquiriendo una estructura orgánica, fluida y descentralizada, que permite responder creativamente a los retos adaptándose eficazmente a unos mercados variables. Así, se forman equipos multidisciplinares coordinados mediante la adaptación mutua (Mintzberg, 1991: 229-257, y 2012). Estas organizaciones serían, aparentemente, más democráticas y participativas. Por su parte, Castells ha aportado la exitosa noción de red, para ilustrar un modelo de organización conformado por nodos interconectados en el que no existe un centro definido (Castells, 2006: 27-30).

La reorganización acometida, desde los años 70, de la actividad laboral y productiva por muchas empresas reflejaba las dificultadas que encontraban las tradicionales estructuras fordistas, diseñadas para la producción masiva de bienes estandarizados, para hacer frente a mercados creciente-

\footnotetext{
4 Sennett ha realizado estudios etnográficos, basados fundamentalmente en entrevista a los empleados y directivos, en empresas como IBM, Goldman Sachs o Lehman Brothers (2006b: 163).
} 
mente diversificados y complejos. Por ello, se habla de postfordismo -término popularizado por Piore y Sabel (1984) — para designar la implantación progresiva de modelos más flexibles de trabajo, mediante la introducción de las nuevas tecnologías y técnicas productivas que favorecen un modo de actividad más fluido y descentralizado. De los trabajadores, se espera en consecuencia que exhiban mayor iniciativa, adaptabilidad y disponibilidad para adquirir nuevos conocimientos (Giddens, 2001: 490-494).

El ritmo del avance tecnológico se ha acelerado en los últimos años - hablándose incluso de una cuarta revolución industrial-, provocando nuevas transformaciones en el proceso productivo y distributivo de bienes y servicios, en la actividad de las organizaciones o en la estructuración del mercado de trabajo. En primera instancia, emerge la amenaza de destrucción de numerosos puestos de trabajo y la acentuación de la desigualdad, alcanzando ahora también al sector administrativo - white collar- ${ }^{5}$. Los contornos de las organizaciones se vuelven aún más difusos, proliferando diversas formas de trabajo flexible, particularmente las modalidades de freelance ${ }^{6}$. Aparecen nuevas oportunidades de empleo, aunque bajo un semblante distinto al que fue habitual. Ciencia, tecnología, ingeniería y matemáticas serían las áreas de conocimiento más demandadas, si bien en general se estimarán ciertas habilidades generales — críticas, contextuales-, no vinculadas a una profesión o función específica (Samans \& Davis, $2017)^{7}$.

Así pues, las investigaciones que desarrolla Sennett enlazan con una constatación bastante extendida, como se acaba de mostrar, acerca de la importancia de los cambios que están atravesando las organizaciones, cuya orientación básica sería la búsqueda de formas más flexibles de producción. Pero, como trata de mostrar, no se trata de un proceso exento de contradicciones, ya que la orientación que sigue se traduce en mayor incertidumbre y precariedad para los afectados.

La desregulación financiera de los años 70 produjo la liberación de grandes sumas de capital, que encauzado a través de fondos de inversión

\footnotetext{
5 Si los avances tecnológicos precedentes afectaron sobre todo a las labores manuales rutinarias, la intensificación de la computarización y el desarrollo de la inteligencia artificial irá afectando previsiblemente a tareas que presentan mayor exigencia cognitiva o capacidad para la resolución de problemas, provocando un desplazamiento en el mercado de trabajo (Frey \& Osborne, 2017).

6 Se trata de una tendencia que refleja un cambio profundo en la forma de trabajar y establecer las relaciones laborales, según el informe sobre el futuro del empleo del World Economic Forum (2016: 17).

La inteligencia artificial, la robotización y los drones, la multiimpresión, los nuevos materiales, la biotecnología y neurotecnología extenderían el potencial sembrado por la digitalización en la Tercera Revolución Industrial con sus extraordinarios avances en tecnologías de la información y la comunicación (Samans \& Davis, 2017: 5).
} 
pasó a controlar numerosas corporaciones. Como resalta Sennett, se trata de un poder ajeno a la cultura establecida en la empresa, y que aspira obtener beneficios a corto plazo $^{8}$. En este contexto, las empresas habían de pugnar por atraer el capital inversor, debiendo mostrar una apariencia de dinamicidad, adaptabilidad e innovación; mientras la solidez o la estabilidad institucional - que remiten a una temporalidad amplia- se convirtieron en factores que generaban una apreciación negativa (Sennett, 2006a: 37-41). En consecuencia, las empresas se embarcaron en procesos periódicos de rediseño de sus estructuras (Sennett, 1999: 3; 2000: 50); tratando de cumplir con dos principios generales vinculados entre sí: el aplanamiento — referido a la estructura del poder - y el acortamiento — referido al horizonte temporal-. Es decir, se eliminan capas burocráticas al tiempo que se contiene la expansión de la base, y las tareas propuestas se circunscriben a un período limitado (Sennett, 2003: 186-190). La flexibilización de la producción va ligada, por tanto, al predominio de una perspectiva cortoplacista, justificada por la inestabilidad de la demanda. Y su implementación en la empresa modifica la distribución interna del poder.

En consecuencia, la tradicional estructuración en niveles superpuestos es reemplazada por una nueva manera de distribuir el poder, la actuación y la toma de decisiones, para liberarse de la rigidez burocrática y convertirse en una organización más ligera, plana, dinámica y flexible (Sennett, 2003: 189-190). El objetivo general es producir bienes más variados cada vez más rápido, logrando una especialización flexible en el funcionamiento interno de la empresa, conforme a la imagen de la red (Sennett, 2000: 5261). Para la realización de objetivos específicos se forman grupos de trabajo que compiten entre sí; y se opta por externalizar algunas funciones, que son desempeñadas por actores ajenos al núcleo tradicional de la empresa - consultorías, subcontratas, plantas de producción u oficinas ubicadas en otros países-. Como resultado, se estaría generando una fragmentación de las distintas operaciones que realiza la empresa, que contrasta con el funcionamiento unificado que propiciaba el modelo piramidal (Sennett, 2006a: 56).

Lo importante es que la profundización en la orientación que dirige el proceso de reordenación institucional le permite a Sennett cuestionar algunas de las aparentes virtudes que se atribuyen al nuevo modelo. Así, sus defensores afirman que el funcionamiento flexible otorga a los distintos miembros de una organización mayor autonomía, capacidad de decisión y actuación, propiciándose una descentralización del poder. En cambio,

8 En ese sentido, recurre al término capital impaciente empleado por Harrison (Sennett, 2006a: 39). 
como afirman diversos críticos del Nuevo Capitalismo, como Sennett o Fleming, la eliminación de capas en la jerarquía y la flexibilización de las relaciones laborales y de producción no se traduce realmente en una mayor distribución del poder, pues de hecho permite que un número más reducido de altos directivos adquiera un mayor control sobre la organización, aunque en ocasiones no conozcan ni sean expertos en la actividad (Sennett, 2000: 49-51; Fleming, 2015: 78-92).

La falta de centralización, pues, sería sólo aparente, tratándose en realidad de una burocracia plana y corta, equivalente a una unidad central de procesamiento. Los avances en las tecnologías de la información permiten incrementar el control desde la dirección, que puede disponer con rapidez de la información sobre lo que sucede en los distintos niveles de la organización (Sennett, 2006a: 41-42), y enviar las órdenes directamente a las diferentes unidades, sin que tengan que ser filtradas a través de las

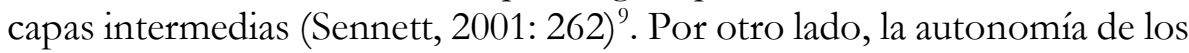
equipos de trabajo sería limitada, pues desde la dirección se les empuja a conseguir los objetivos marcados. En definitiva, la flexibilización organizativa no propiciaría una mayor igualdad o participación, sino que antes bien da lugar a nuevas formas de poder y control, tratándose de una dominación sin forma definida, más opaca, desigual e intensa (Sennett, 2000: 49-58). Se abre, por tanto, una brecha entre el centro, desde el que se controla y se toman las decisiones, y la periferia, en la que los subordinados carecen de las herramientas necesarias para integrarse activamente en la ordenación institucional.

$\mathrm{El}$ interés que desde una perspectiva filosófica ofrece el análisis de Sennett radica en que trata no sólo de dar razón de la emergencia de una nueva forma de organizar la actividad productiva, pues en la medida en que incita al lector a valorar cuál es la orientación que la dirige, se plantea la necesaria exigencia crítica de cuestionar los principios y valores que rigen las tendencias económicas e institucionales dominantes en la actualidad. A nuestro juicio, lo que presenta de distintivo y original el análisis del autor estadounidense es su capacidad para profundizar en las consecuencias que presentan estas transformaciones en la vida de las personas y en las relaciones sociales, y en cómo pueden responder a las características concretas y posibilidades que ofrece el marco social-institucional en el que viven y actúan. La profundización, que se realiza a continuación, en las

Esta forma de tratar con la información le sugiere a Sennett la imagen de un disco o CD (2003: 189) o de una rueda (2001: 330), para ilustrar el nuevo modelo organizativo. 
nociones de carácter y artesanía que emplea Sennett permitirá definir de manera más precisa la originalidad de su propuesta ${ }^{10}$.

\section{LA CORROSIÓN DEL CARÁCTER EN EL CONTEXTO ACTUAL}

La noción de carácter es una de las más fructíferas en el pensamiento de Sennett, siendo especialmente trabajada por este autor a partir de los años 90, en relación precisamente con el análisis de las transformaciones que están produciéndose en el mundo de las organizaciones. Pero, además, sería adecuado, como muestra un estudio profundo de su obra, contemplarla dentro de su concepción general del ser humano, en la que el trabajo se revela como una fuente primordial para una elaboración narrativa de la identidad (Muñoz Miralles, 2014). Pues el interés primordial que ofrece la obra de Sennett para una ética empresarial atenta a los retos del presente reside justamente en que sitúa en el foco a un ser humano entendido como participante social e institucional competente, que se ve afectado por los procesos de transformación. Desde esta perspectiva, resalta el valor que tiene para los individuos el poder dar sentido a su actuación en el mundo laboral, lo que realizan trazando una historia personal. Sin embargo, cuando su realización es obstaculizada por las condiciones materiales existentes, se ve afectada la estimación de la propia valía y, como resultado, la biografía personal se resquebraja (Smith, 2007: 202).

La posibilidad de elaborar relatos vitales fundamentados en la experiencia laboral dando lugar a una identidad personal integrada, se antoja cada vez más complicada, debido a que el sistema está dejando de ofrecer las certezas que ayudaban a planificar el futuro y ordenar la experiencia (García Ferrer, 2016) ${ }^{11}$. Paralelamente, la sostenibilidad de las relaciones sociales se ve mermada por el dominio de una perspectiva cortoplacista. Es en este sentido en el que hay que entender que Sennett hable de una corrosión del carácter ${ }^{12}$ como efecto de la extensión del Nuevo Capitalismo.

10 Siguiendo la categorización de crítica propuesta por Boltanski y Chiapello (2002), la propuesta de Sennett no sólo es reformista sino que tiene un componente radical. Su pretensión, tras el análisis profundo de la obra de R. Sennett, no es simplemente desvelar una situación de injusticia para que el capitalismo ofrezca una respuesta correctiva a tal situación creada, sino más bien proponer un sistema distinto de relaciones y por tanto alternativo al existente.

11 Fleming considera, como Sennett podría suscribir, que la situación del trabajo en la actualidad supone un retorno a las condiciones iniciales del capitalismo, de tal modo que más que de prosperar, se trata ahora de lidiar diariamente con una precariedad establecida (Fleming, 2015: 4-5 y 198).

12 El concepto de carácter cuenta con una larga historia en el pensamiento filosófico y social, siendo de especial relevancia su relación con el proceso de formación de la persona como un ser moral, pues, como señala Aranguren, se trata del modo de ser adquirido 
La comprensión del carácter que propone Sennett es deudora, de manera más específica, de la definición empleada por Gerth y Mills, y depende de una determinada concepción de la sociedad que se formaría a partir del encuentro entre extraños (Sennett, 2003: 62). Desde esta perspectiva, el carácter podría considerarse como el "aspecto relacional de la personalidad", entendiendo que es posible mantener vínculos emocionales más allá del limitado círculo de las relaciones cara a cara, identificándose así con la capacidad de implicar un mundo más amplio en la vida personal (Sennett, 2003: 63-64). Según Mesienhelder (2006), la propuesta de Gerth y Mills permite reintroducir una aproximación más estructural a la noción de carácter, que representa la intersección entre la persona y la sociedad (Meisenhelder, 2006: 61- 62). Así recalcan que la persona se forma en un contexto institucional, al interiorizar los diversos roles, las expectativas ligadas a ellos y las motivaciones para su cumplimiento (Gerth \& Mills, 1984: 163ss).

Sennett, subraya las virtualidades comunicativas del concepto de carácter donde éste sería la dimensión de la persona cuyo óptimo desarrollo le permite dejar atrás una imagen de sí mismo limitada por el autointerés egoísta o por una identidad construida sobre la semejanza, introduciéndose en las complejidades propias de la sociedad más amplia, atendiendo a sus reglas y exigencias, y comprometiéndose con su funcionamiento (Sennett, 2003: 63).

Por otro lado, Sennett incide en la contraposición entre el concepto tradicional de carácter frente al más moderno de personalidad, entendiendo que ésta se referiría más bien a los deseos y sentimientos íntimos, mientras el primero tendría un alcance más amplio, subrayando más nítidamente los aspectos interrelacionales de la vida personal, e incluyendo las dimensiones ética y temporal. Así, según afirma este autor, el carácter tiene que ver con aquellos rasgos que esperamos que los demás aprecien en nosotros porque los consideramos valiosos, por lo que tratamos que sean sostenibles en el tiempo. Por ello, remite a la continuidad de nuestra experiencia emocional, en la medida en que nos vincula a los otros, expresándose en la lealtad y el compromiso, y relacionándose con el valor ético que otorgamos a nuestros deseos y relaciones (Sennett, 2000: 10). De esta forma, cabe incidir en la importancia de la dimensión institucional de la vida per-

por uno mismo a través de su vida, constituyendo una especie de segunda naturaleza (Aranguren, 2003: 6-10). Este concepto contiene, por tanto, una dimensión ética fundamental, refiriéndose al modo de ser que uno se va forjando a través de sus elecciones. En ese sentido, la ética es el saber que nos orienta en la forja de un buen carácter (Cortina, 1994: 18-19). 
sonal para la formación del carácter. Como ha percibido Hartman, las cualidades que otorgan fortaleza al carácter, y que permiten a una persona asumir y mantener sus compromisos, refuerzan la solidez de las organizaciones (Hartman, 1998: 551). Y se distinguirían, precisamente, por estar orientados "a largo plazo" (Sennett, 2000: 29).

Cabe resaltar, a nuestro juicio, la vinculación entre la orientación ética presente en el pensamiento de Sennett y su insistencia en la importancia de una temporalidad amplia, cristalizando nítidamente en la noción de $c a-$ rácter. Su consolidación requiere de tiempo para poder desplegar la dedicación y el esfuerzo necesarios, asentando la confianza que permite establecer relaciones sólidas con el entorno. Cuando prima el presentismo o la urgencia, y se carece de certezas, en cambio, las cualidades morales se resienten y, en consecuencia, el carácter se debilita.

Para Sennett la formación del carácter es fruto de un proceso dinámico de aprendizaje, a través del cual las antiguas creencias y los hábitos establecidos van transformado su significado y en el que la confianza se ve modificada. La consecución de un carácter sólido requiere del sostenimiento de una sensación básica de la autoconfianza —o seguridad ontológicacon la que afrontar los retos que plantea el entorno social e institucional en que nos desenvolvemos. La curiosidad impulsa al individuo a interesarse por asuntos que trascienden su esfera íntima, poniendo a prueba las certezas previas, explorando nuevas formas de relación con los otros (Sennett, 2003: 231-245).

Pero Sennett advierte de la dificultad de establecer una narrativa personal careciendo de anclajes emocionales y experienciales en un recorrido laboral y vital que se presenta fragmentado e incierto. De suerte que, por un lado, la orientación al corto plazo actúa en la dirección opuesta a la formación de un carácter, que como se acaba de afirmar, requiere de una perspectiva temporal mucho más amplia. La precariedad en el empleo, el declive de las carreras laborales, o la provisionalidad de los equipos de trabajo, difícilmente podrían propiciar el florecimiento de cualidades como la lealtad o el compromiso (Sennett, 2000: 28-31). Y, por otro lado, el nuevo régimen irradia indiferencia, desgastando los vínculos sociales por los que se promueve la reciprocidad, el cuidado, la dependencia y el apoyo mutuos, e impidiendo la elaboración de una narrativa compartida ante las dificultades. La sensación de aislamiento social obstruye el despliegue de una percepción adecuada de nuestras capacidades sociales, de confiablidad e importancia para los demás, y en definitiva, de la constancia personal sobre la que sostiene el carácter (Sennett, 2000: 152-155).

Sennett se esfuerza en desvelar el aspecto engañoso que presenta el nuevo modelo institucional, poniendo de manifiesto su relación contradictoria con el carácter. Aparentemente, invita a los individuos a romper 
con los hábitos establecidos, a no abandonarse a la sensación de seguridad, a explorar activamente las novedades que se suceden en el mundo actual. Sin embargo, denuncia el autor estadounidense, no se estimula verdaderamente la curiosidad o la iniciativa, sino que se fuerza a la gente a cambiar su situación, aunque las posibilidades reales de intervención son mucho más limitadas de lo que se promete. Las decisiones de cambio se imponen desde arriba, y la mayoría de la gente se ve forzada a asumir riesgos que superan su capacidad de acción y control, realizando el giro hacia la sociedad sin disponer de suficientes recursos o de una densa red de contactos para poder moverse con ciertas garantías en las circunstancias que impone el Nuevo Capitalismo (Sennett, 2003: 240-244). Las nuevas formas de organización del trabajo impiden el aprendizaje, ya que la premura por los resultados y la provisionalidad no permiten obtener un conocimiento apropiado de los demás, aprender de los errores, o atender adecuadamente a los problemas (Sennett, 2006a: 112).

En definitiva, Sennett nos invita a pensar que el Nuevo Capitalismo más que liberar, deja a los individuos desprotegidos, restándoles capacidad efectiva de participación, y desestimulando la activación de sus capacidades sociales. No ofrece, por tanto, un marco propicio para el despliegue del carácter ni para la elaboración de un relato biográfico integrado narrativamente, en el que la identidad laboral ocupe un lugar clave. Por ello este autor considera vital reivindicar el potencial que tiene el trabajo para el desarrollo humano, lo que realiza a través de la noción de artesanía. Concepto que, como se tratará de argumentar, será una pieza importante para el desarrollo en el contexto actual de la ética empresarial.

\section{LA ARTESANÍA: EL DESPLIEGUE DEL CARÁCTER A TRAVÉS DEL TRABAJO}

La participación en el mundo del trabajo constituye, siguiendo los escritos de Sennett, un ingrediente fundamental de la realización personal. Pero los cambios que están produciéndose en ese ámbito cuestionan la posibilidad de desplegar una trayectoria laboral integrada, ya que la flexibilización de la producción va acompañada de una precarización del trabajo $^{13}$. Ante esta situación, Sennett reivindica la importancia de fortalecer una concepción profesional de la vida laboral de los individuos, no sólo como parte de su propio desarrollo como seres autónomos, ya que la solidez y confiabilidad de las instituciones se alimenta de unos miembros

\footnotetext{
13 Esta tesis también es mantenida por Boltanski y Chiapello (2002) así como por Fleming (2015), este último además afirma que no sólo es la flexibilización sino también las políticas de subsidio, promovidas por las economías y políticas neoliberales, las que están ayudando a mantener tal precarización.
} 
bien formados e integrados en su actividad. La exploración de las posibilidades que ofrece la noción de artesanía es el camino escogido por Sennett para tratar de responder a la fragmentación de la experiencia laboral que genera la nueva economía.

En tanto se considere la artesanía como forma de actividad productiva, se trata de dilucidar en qué medida la propuesta de Sennett sirve para denunciar las deficiencias del nuevo modelo económico e institucional, reivindicando una idea más ajustada a unas potencialidades humanas que, lejos de fomentarse, se estarían viendo mermadas en el contexto actual. Así, pese a que es común hablar hoy en día de una economía de las habilidades, este autor ofrece una reflexión sobre el papel real que concede la sociedad actual a la posesión de la habilidad, contrastándolo con la noción de artesanía, que no encuentra la apreciación que merecería. Por ello, Sennett se esfuerza en explicitar su virtualidad para el desarrollo económico, social, y también personal, lo que invita a resaltar, a nuestro juicio, la fuerza moral que le es inherente. Pues, según trata de demostrarse aquí, el valor que ofrece el enfoque de Sennett sobre la artesanía radica en que no se limita a definir una forma de actividad productiva, sino que propone un modelo formativo integral del ser humano, vinculándose así estrechamente a la noción de carácter.

Se trata de entender la artesanía como una práctica, es decir, una forma de hacer cosas y de relacionarse con los otros, un modo de actuación socialmente significativa, dinámico y abierto, cuya realización implica un aprendizaje para los participantes que impulsa a su vez su revisión y perfeccionamiento (Calhoun \& Sennett, 2007: 6). Respondería así a una comprensión pragmatista de la experiencia, tratándose no meramente de una vivencia subjetiva, sino sobre todo de una acción o relación que reclama la participación activa del individuo (Sennett, 2009: 353-356) ${ }^{14}$. La artesanía supone, por tanto, un modelo de realización personal mediante la inserción en prácticas regladas socialmente que implican un trato activo con los otros $^{15}$.

14 Früwald destaca la importancia de la noción de experiencia para comprender mejor las propuestas de Sennett, desde su vinculación al neo-pragmatismo. De esta manera, aparecen interpenetrados lo físico y lo mental, entendiendo que existe una equivalencia funcional entre nuestra capacidad para dar forma a las cosas y la que empleamos en nuestras relaciones sociales (Früwald, 2009: 40). Y es que para Sennett la artesanía designaría un impulso humano fundamental, el de realizar bien una tarea (Sennett, 2009: 38).

15 Tallis observa que la actividad manual, en tanto establece una relación activa con el mundo, permite adquirir conciencia y expresar el sentido de la agencia personal, incorporando además una dimensión social susceptible de realizarse cooperativamente (Tallis, 2003: 231-233 y 260). 
El modelo artesanal que propone Sennett, por tanto, no consiste meramente en un esquema formativo para la adquisición y perfeccionamiento de unas habilidades técnicas o actitudinales que permitan delinear una trayectoria profesional consistente; ofrece un camino para el despliegue de las cualidades relacionales y sociales del carácter, mediante el fomento de las disposiciones favorables a la cooperación. Es decir, el trabajo y la sociabilidad se nutrirían de un fondo común que es posible ir desplegando conjuntamente. De esta manera, sería posible superar la escisión planteada por Arendt entre técnica y razón práctica, entre el ser humano como trabajador y como productor de vida en común (Sennett, 2009: 11-20). La comprensión que ofrece Sennett del ser humano como homo faber no se restringe al trabajo material, afirmando más ampliamente que "el Hombre es productor de sí mismo, creador de la vida por medio de prácticas concretas" (Sennett, 2012: 11). Es decir, el ser humano tiene la virtualidad para formarse a sí mismo a través de las prácticas sociales en las que participa. En ese sentido, el trabajo ofrece un componente social de gran valor para el despliegue del carácter, ya que la mayor parte de tareas requieren del esfuerzo coordinado de varias personas.

La idea de artesanía en Sennett apuntaría, pues, hacia un horizonte más amplio que el que parecería aportar a primera vista el mero trabajo manual, revelándose a nuestro juicio como una vía privilegiada para la formación del carácter. En ese sentido, cabe observar, por un lado, que se identifica como una tendencia duradera, y que la realización correcta de un trabajo exige amoldarse a unos patrones objetivos de excelencia (Sennett, 2009: 20).

Pero el logro de la excelencia es fruto de un largo proceso de aprendizaje, que requiere de dedicación y compromiso, cualidades que han de ser durables. Sennett observa que el aprendizaje de una tarea impone un ritmo determinado, mediante el surgimiento de problemas concretos y la exploración de las posibles soluciones, estableciéndose un diálogo entre la práctica y el pensamiento que se solidifica en forma de hábito (Sennett, 2009: 21). Cabe recordar que para Aristóteles el hábito era el reflejo en forma de acción de una actitud firmemente interiorizada, tratándose de una disposición permanente a actuar de determinada manera, manifestando así la excelencia del carácter. La adquisición de un hábito requiere de esfuerzo y práctica continuada, como ocurre en el aprendizaje de cualquier habilidad técnica (Conill \& Montoya, 1985: 130-134).

A nuestro juicio, en el planteamiento aristotélico se muestran algunos de los elementos que definen la noción de artesanía propuesta por Sennett, que trasciende la mera dimensión técnica, comprendiendo el desarrollo del carácter como un proceso de aprendizaje prolongado, que requiere de una actitud comprometida con el logro de la excelencia a través del esfuerzo y 
la constancia. En ese sentido, cabe entender que los hábitos ayudan a forjar un carácter artesanal, siendo imprescindibles para la consolidación del dominio de una determinada habilidad. Se trata de cualidades de enorme valía para unas organizaciones que aspiren a la confiabilidad y continuidad de sus actuaciones de cara a la sociedad.

Sennett precisa que la posesión de una habilidad específica se concibe como una práctica adiestrada, es decir, que alcanzar su dominio exige de un ejercicio continuado y rutinizado (Sennett, 2009: 53-54). La excelencia en la acción, como también habría sostenido Aristóteles, no brota de manera natural o espontánea, sin esfuerzo, sino que debe ejercitarse, siendo preciso volver repetidamente sobre ella hasta que se consolide en forma de hábito.

Sennett, por su parte, añade que enfrentarse creativamente a las resistencias que presenta la realidad material proporciona a la larga un mejor conocimiento de ella, mediante la exploración de soluciones alternativas a los problemas planteados, aprendiendo a manejar positivamente la frustración que inicialmente provoca. El buen artesano, sería, por tanto, el que ha aprendido a manejar productivamente la problematicidad (Sennett, 2003: 238-242; 2009: 263-278). Y, por este camino, habría que añadir, se habría ido forjando un carácter sólido.

La idea de artesanía le permite a Sennett, además, indagar en las virtualidades del trabajo entendido como actividad eminentemente social, que se emprende de manera cooperativa, desplegando las capacidades sociales y comunicativas de los seres humanos (Sennett, 2012). Resaltar la dimensión social del trabajo y sus virtualidades formativas para el carácter supone trascender una consideración meramente instrumental del mismo. Ya Marx había apreciado que el trabajo no debía contemplarse como un medio, ya que se trata de una actividad social formativa de las cualidades humanas (Sayers, 2007: 432-441) ${ }^{16}$. La originalidad del tratamiento que propone Sennett de la idea artesanal, según estamos exponiendo, consiste en describirla como un modelo de realización humana, que halla en la práctica abordada socialmente e institucionalmente integrada, el camino más adecuado para la realización del carácter.

Desde esta perspectiva, la formación completa del ser humano requiere superar las limitaciones de la subjetividad involucrándose en un conjunto de reglas y expectativas sociales, modelando y perfeccionando su modo de actuar a lo largo del tiempo. Pero la fragmentación de la experiencia propiciada por una economía dominada por el presentismo supone

16 Si bien, si se entiende la artesanía como forma de producción manual, para Marx se revelaría como limitada en su alcance e incompleta en su función formativa (Sayers, 2007: 439-450). 
el desafío más acuciante en nuestros días para la viabilidad de un proyecto artesanal de desarrollo humano.

\section{LA ARTESANÍA EN EL MARCO DEL NUEVO CAPITALISMO: ORIENTACIO- NES ÉTICAS}

La idea de artesanía propuesta por Sennett permite denunciar las limitaciones que impone la nueva economía al desarrollo de las posibilidades laborales y de las capacidades sociales y narrativas de los individuos. Pero se trata de valorar hasta qué punto ofrece una respuesta adecuada y viable a los retos institucionales del presente.

En el pasado el modelo de organización burocrática ofrecía un marco definido para el desarrollo de unas historias laborales unificadas en forma de carrera. El desempeño de un oficio actuaba como una fuente primordial de sentido para la vida de las personas. Sin embargo, distaba de ser el marco ideal para tanto la realización personal como para el fomento de una concepción artesanal del trabajo. Por ello autores diversos ya habían denunciado que la burocracia y el sistema industrial fordista propiciaban un funcionamiento anquilosado en las rutinas, que dejaba un margen muy escaso para la iniciativa personal, que resultaba poco motivador, no ofreciendo un reconocimiento personalizado a sus empleados, pero fomentando su acomodación (Giddens, 2001: 448ss. y 490; Sennett, 2000: 40ss.; Ruiz Olabuénaga, 1995: 163-167).

Los sectores punteros de la economía - siguiendo con la argumentación de Sennett- estarían hoy actuando como vanguardia a la hora de implementar nuevas técnicas y formas de organizar el trabajo que, según sus defensores, permiten liberarlo de las rigideces que imponía el modelo tradicional, otorgando a los individuos mayor margen de iniciativa y responsabilidad, ofreciendo nuevas posibilidades de empleo y promoción, gracias al dinamismo y la flexibilidad de la nueva economía ${ }^{17}$. Pero Sennett se esfuerza en desvelar lo engañoso de estas presunciones, apuntando a los efectos corrosivos de la misma. Así, la supuesta facilitación de la iniciativa personal más bien sería una manera de justificar la eliminación de garantías materiales y legales que conlleva la celebración del riesgo, imponiendo en realidad una creciente desigualdad de oportunidades (Sennett, 2003: 243-245). De manera similar, Beck observó que el aumento del paro y la introducción de nuevas modalidades de ocupación, más flexibles y

\footnotetext{
17 En ese sentido, es significativo que Toffler considerara que la desestandarización de la producción propiciada por los avances tecnológicos impulsaba una nueva forma de artesanía, acorde a los requerimientos de un consumo individualizado (Toffler, 1984: 7172 y 116).
} 
plurales, excluyen la viabilidad del empleo estable de por vida, extendiéndose una amplia zona gris de subempleo (Beck, 1998: 178 ss.).

La precarización del empleo propiciada, en buena medida, por la urgencia del beneficio parece dibujar un escenario poco favorable para el desarrollo de una carrera profesional estable y, por tanto, para el despliegue de las cualidades artesanales. En ese sentido, Sennett recalca que las tendencias actuales alteran la forma de considerar la capacitación profesional de los trabajadores y las posibilidades reales de servirse de sus conocimientos y aptitudes. La planificación de la producción orientada a dar respuestas rápidas a las demandas cambiantes del mercado, o la flexibilización de las legislaciones laborales entre otros factores, explicarían que el mundo del trabajo presente hoy un aspecto altamente fragmentado, en el que abunda la temporalidad, la subcontratación, la flexibilidad horaria, o el trabajo por cuenta propia.

El nuevo modelo propone, en relación a ello, una redefinición de la habilidad que la aleja, según denuncia Sennett, de la noción artesanal, ya que comporta una desvalorización de la experiencia acumulada en favor de la potencialidad y el dinamismo que se atribuye a los jóvenes. La defensa del talento potencial implica, según este autor, el uso de unos criterios imprecisos para juzgar la valía personal, ya que se dirigen hacia un objeto aún indefinido, desdeñando los logros concretos que se muestran a través del trabajo como resultado del esfuerzo y la práctica sostenida en el tiempo. En lugar de la posesión de una habilidad específica, susceptible de ser perfeccionada, se apuesta por habilidades portátiles, premiando la capacidad para aprender cosas nuevas, para reciclarse permanentemente, para poder realizar así tareas sin conexión aparente entre sí, adaptándose a diferentes requerimientos según las circunstancias del momento (Sennett, 2006b). Y, según se ha señalado más arriba, las tendencias de futuro parecen afianzar esa dirección hacia una redefinición radical de la habilidad y del empleo.

La promoción de un tipo de habilidad potencial y portátil se aviene, ciertamente, con los principios que inspiran la reordenación institucional acometida por esas organizaciones de vanguardia. Pero la pretensión de lograr un funcionamiento flexible y adaptable, junto al objetivo de obtener un rendimiento a corto plazo, desconectan la actividad organizacional en el presente tanto de la historia de la propia corporación como de una visión amplia del porvenir. Ese presentismo explica su desdén por el valor de la experiencia acumulada o el compromiso con la función desempeñada, cualidades vinculadas a la definición de oficio (Sennett, 2009: 31-33 y 349).

En estas condiciones, la relación de los trabajadores con las empresas se vuelve más endeble. Lo más habitual hoy en día para cualquier individuo 
es recorrer un camino laboral incierto, pasando de un empleo a otro, teniendo que recurrir a destrezas variadas sin poder profundizar en ninguna habilidad específica, y permaneciendo en una situación de precariedad continua (Sennett, 2009: 326). La posibilidad de desarrollar una carrera profesional no encuentra ya respaldo en un marco institucional que elude la visión a largo plazo y corroe las virtudes asociadas a una temporalidad amplia, como el compromiso o la lealtad. El desarrollo de un oficio, en definitiva, deja de ser un camino viable para desplegar una historia laboral (Sennett, 2009: 49-52).

Una formación de tipo artesanal parece no encajar bien en un mercado laboral fragmentado y en una economía flexible y dinámica que aboca a los individuos a someterse a una compleja convivencia con el cambio y el riesgo permanentes. La artesanía propone, en principio, un modelo de realización personal que se nutre del perfeccionamiento orientado de una pericia técnica específica, lo que requiere del respaldo de un marco institucional estable y comprometido. En cambio, la realización de una aspiración humana fundamental, como es la de dar sentido a la experiencia, parece tropezar con dificultades insalvables especialmente en un ámbito fundamental para forjar una autocomprensión personal sólida y coherente como es el mundo del trabajo.

Por ello, se trata de explorar respuestas a los retos del futuro que resulten viables en su aplicabilidad, aunque sin perder de vista su contenido moral y las expectativas legítimas de los afectados. Desde el Foro Económico Mundial se subraya la urgencia, ante el surgimiento de nuevas especialidades y tareas que patentizan el desfase de muchos conocimientos y procedimientos afianzados, de acometer una actualización permanente de las destrezas. Para ello, proponen un enfoque interdisciplinar de la formación que potencie no sólo los conocimientos específicos - condicionados por el ritmo de avance tecnológico- sino especialmente las aptitudes generales ${ }^{18}$. Se requeriría la implicación tanto de los gobiernos como de las empresas y de los propios individuos para anticipar y afrontar las demandas y oportunidades que plantea el complejo mundo laboral del siglo XXI (World Economic Forum, 2016: 25-32), por tanto, de una auténtica gobernanza orientada éticamente en sentido cosmopolita (González-Esteban, 2013).

\footnotetext{
18 Habilidades cognitivas como el razonamiento matemático y lógico, habilidades sociales, la capacidad para la resolución de problemas, o el manejo de las tecnologías de información y comunicación serían destrezas valiosas en cualquier ámbito profesional (World Economic Forum, 2016).
} 
La respuesta que pueda ofrecer el proyecto artesanal de Sennett debe ir más allá de una reforma de los procedimientos educativos que esté dirigida por las demandas del mercado - aunque, por supuesto, incorporándolas como un componente destacado-, encuadrándose en una comprensión formativa integral del ser humano. En ese sentido, señala la importancia de que tanto las instituciones dedicadas a la enseñanza como las propias organizaciones empresariales apuesten por una visión de la formación individual comprometida con el fomento de las vocaciones, de manera que puedan cumplirse en un desarrollo adecuado de las habilidades ajustándose a los retos del presente. Así, considera que la inversión de las empresas en la formación de sus empleados en habilidades sucesivas, mediante el reciclaje laboral, manteniendo un espíritu artesanal, permitiría responder a las exigencias actuales del mercado - en cuanto a competencia, especialización o variabilidad-, además de aportar beneficios a largo plazo a las empresas, los trabajadores y el conjunto de la sociedad (Sennett, 2009: 326-328). Sin embargo, estas sugerencias interesantes no son profundizadas por el autor.

Aunque, según se ha expuesto, Sennett manifiesta sus recelos ante la preferencia que detecta en las empresas de vanguardia hacia unas habilidades más generales o ubicuas - en forma de talento potencial o como habilidades portátiles- en detrimento de una formación profesional específica continuada, que estaría en el núcleo de la idea artesanal, podría argüirse que el escenario económico y laboral que está configurándose parece requerir una reconsideración de la misma. En cualquier caso, Sennett trata de evitar abandonarse a una mirada resignada o nostálgica ${ }^{19}$. Y, a nuestro juicio, puede hallarse precisamente en el modelo de formación personal artesanal que proporciona Sennett la base sobre la que elaborar una definición más amplia —es decir, no restringida a una comprensión estrictamente profesional - de la artesanía, que se revelaría además más ajustada a los retos que plantea ya el futuro.

19 Estas posiciones son las que se esconden, a nuestro juicio, tras las propuestas que Boltanski y Chiapello (2002: 441ss) que apuestan por una recuperación de la "crítica artista" - demanda de autonomía, creatividad, autenticidad y liberación- para lograr que la "crítica social" — solidaridad, seguridad e igualdad- sea posible; o la mantenida por Fleming (2015: 188ss) al apostar por una autoabolición de la clase de trabajo global e interdependiente mediante el abandono de la subordinación que genera el trabajo. Sennett, sin embargo, aprecia que el resultado del ataque de la Nueva Izquierda al sistema burocrático a la postre se ha revelado paradójico, pues su desmantelamiento ha resultado en favor de un capitalismo flexible en el que la liberación individual y social es ficticia (Sennett, 2003: 258-262). 
La clave respecto al futuro de la artesanía reside, pues, en la ordenación institucional. Hay que resaltar que la artesanía ofrece algunas cualidades que precisa cualquier organización cuyo propósito esté vinculado a la duración en el tiempo, y que haga suyos valores como la solidez, la estabilidad o la confiabilidad. Como observa Román Maestre, la posibilidad de desplegar una carrera profesional permite realizar en el trabajo unos criterios de comportamiento profesional y personal, que reflejan un sentido de la responsabilidad, cualidades básicas para que se pueda ir consolidando un ethos corporativo que defina un proyecto empresarial sólido y confiable (Román Maestre, 2004: 10). En ese sentido, puede constatarse un creciente interés en las empresas por forjarse un carácter corporativo virtuoso a través de su actuación cotidiana cristalizando en forma de hábitos (Chun, 2005: 272).

Para situar adecuadamente esa tarea de diseñar instituciones comprometidas con su permanencia y estabilidad y propicias para un desarrollo de sus miembros que esté orientado artesanalmente, es necesario aportar un marco crítico bien definido como se viene proponiendo desde la ética económica y empresarial en las últimas décadas (Cortina et al., 1994; Conill, 2003 y 2004; García-Marzá, 2004 y 2013; González-Esteban, 2013).

La empresa bien organizada, cuyo horizonte remite a una temporalidad amplia, sabe valorar las aportaciones de unos trabajadores estables, responsables y bien formados, que pueden integrar las virtudes propias de la actividad artesanal en el funcionamiento cotidiano de la empresa, obteniendo de esta manera una mayor lealtad e implicación de sus miembros, así como su esfuerzo continuado y disciplinado en la búsqueda de la calidad en su trabajo, fruto de su compromiso fundamental con su oficio. Se trata de un tipo de bienes que difícilmente pueden obtenerse de trabajadores temporales o externos, formados de manera fragmentaria o limitada, poco motivados, y escasamente comprometidos con una organización que simplemente los instrumentalice. En este sentido es necesaria una consideración más amplia del concepto de bien (Ramis Olivos, 2014) y seguir trabajando en esta propuesta artesanal que luche contra la corrosión del carácter en las organizaciones desde horizontes éticos compartidos.

\section{VALORACIÓN DE LA PROPUESTA DE SENNETTT}

Sennett ofrece un análisis de notable interés acerca del escenario organizativo y laboral que está perfilándose en nuestro tiempo, que sitúa en el foco de la reflexión al individuo que ve alteradas las posibilidades de realización de sus capacidades laborales, narrativas y sociales. No obstante, no pueden obviarse las limitaciones de su planteamiento, que a nuestro 
juicio no acaba de definirse como una denuncia, una propuesta de reforma, o una alternativa viable y consistente. Si bien, a nuestro juicio, frente a propuestas como las de Fleming que invitan a trascender radicalmente el capitalismo, Sennett propondría una reforma profunda del sistema vigente mediante la incorporación de valores y principios que devuelvan un mayor grado de justicia, inclusividad y participación a las relaciones institucionales.

No obstante, habría que señalar la falta de concreción de su propuesta a la hora de plasmar la aplicabilidad del modelo artesanal a la realidad del mundo institucional, echándose a faltar una definición mejor articulada. Hay que apreciar el esfuerzo por revalorizar el trabajo como actividad formativa del carácter, como una práctica social, apuntando a la necesidad humana de reintegrar una experiencia que se presenta hoy dispersa y fragmentada, en un relato vital coherente y comprensible. Sin embargo, queda como tarea pendiente indagar cuáles son las claves que permitan recomponer las relaciones sociales y laborales, así como las capacidades para elaborar esa narrativa experiencial en el actual contexto de precariedad y cambio permanente. Las reflexiones de Sennett, sin duda, muestran los límites y contradicciones de la organización del trabajo, haciendo patente la necesidad de repensar su función en el mundo actual.

Desde la perspectiva de la ética empresarial, es preciso ofrecer respuestas a los retos que se están planteando, siendo una de sus tareas definir un modelo de empresa comprometido con los fines que le son propios y con la sociedad, capaz de generar confianza para constituirse como un proyecto sólido en el tiempo. La idea de una formación artesanal de los trabajadores puede actuar como orientación adecuada para tal propósito, abordando la imprescindible revalorización del componente humano de la actividad económica e institucional. Pero tales propósitos se antojan complicados si globalmente no se adoptan las políticas necesarias para reconducir la actividad económica y comercial en una dirección más inclusiva, estable y duradera, liberándose de la hegemonía de la lógica financiera cortoplacista e irresponsable que domina en la actualidad.

\section{REFERENCIAS}

-Albert, M. (1991). Capitalismo contra capitalismo. Buenos Aires: Paidós.

-Albert, M. \& Gonenc, R. (1996). The future of Renish capitalism. The Political Quarterly, 3(67), 184-193.

-Aranguren, J. L. (2003). La ética y su etimología. Arbor, 174(687-688), 591-606.

-Beck, U. (1998). La sociedad del riesgo. Hacia una nueva modernidad. Barcelona: Paidós.

-Boltanski, L. \& Chiapello, È. (2002). El nuevo espíritu del capitalismo. Madrid: Akal. 
-Calhoun, C. \& Sennett, R. (2007). Introduction. En C. Calhoun y R. Sennett (eds.), Practicing culture (pp. 1-12). London-New York: Routledge.

-Castells, M. (1996). The Rise of Network Society. Oxford: Blackwell.

-Castells, M. (2006). La Sociedad red: una visión global. Madrid: Alianza.

-Chun, R. (2005). Ethical Character and Virtue at Organizations: An Empirical Assessment and Strategic Implications. Journal of Business Ethics, 57, 269-284.

-Conill, J. (2003). El sentido de la economía en tiempos de globalización. Daimon. Revista de Filosofía, (29), 9-15.

-Conill, J. (2004). Horizontes de economía ética. Aristóteles, Adam Smith y Amartya Sen. Madrid: Tecnos.

-Conill, J. \& Montoya, J. (1985). Aristóteles: sabiduría y felicidad. Madrid: Cincel.

-Cortina, A., J. Conill, A. Domingo \& D. García-Marzá (1994). Ética de la empresa. Madrid: Trotta.

-Crouch, C. (2005). Models of Capitalism. New Political Economy, 10(4), 439-456.

-Ebbinghaus, B. \& Manow, P. (2001). Studying varieties of welfare capitalism. En

B. Ebbinghaus y P. Manow (eds.), Comparing welfare capitalism: Social policy and political economy in Europe, Japan and the USA (pp. 1-26). London: Routledge.

-Fleming, P. (2015). The Mythology of Work. How capitalism persits despite itself. London: Pluto Press.

-Frey, C. B. \& Osborne, M. (2017). The future of jobs. How susceptible are jobs to computerisation? Technological Forecasting and Social Change, (114), 254-280.

-Frühwald, W. (2009). Laudatio in honor of Richard Sennett (Gerda Henkel Prize). En How I Write: Sociology as Literature, Award of the Gerda Henkel Prize 2008 (pp. 37-45). Münster: Rhema.

-García-Marzá, D. (2004). Ética de la empresa. Del diálogo a la confianza. Madrid: Trotta.

-García-Marzá, D. (2013). La dimensión ética del diseño institucional. En E. González-Esteban (ed.), Ética y gobernanza: un cosmopolitismo para el siglo XXI (pp. 31-58). Granada: Comares.

-García Ferrer, B. (2016). La constitución de la identidad personal en el nuevo capitalismo y sus repercusiones morales. Daimon. Revista de Filosofía, (67), 117131.

-Gerth, H. \& Mills, C. W. (1984). Carácter y estructura social. La psicología de las instituciones sociales. Barcelona: Paidós.

-Giddens, A. (2001). Sociología. Madrid: Alianza.

-González-Esteban, E. (2013). De la gobernanza política a la gobernanza cosmopolita. En E. González-Esteban (Ed.), Ética y gobernanza: un cosmopolitismo para el Siglo XXI (pp. 3-30). Granada: Comares.

-Hall, P. \& Gingerich, D. W. (2004). Varieties of Capitalism and Institutional Complementarities in the Macroeconomy: An Empirical Analysis. British Journal of Political Science, 39(3), 449-482.

-Hartman, E. M (1998). The role of character in business ethics. Business Ethics Quarterly, 8(3), 547-559.

-Meisenhelder, Th. (2006). From character to habitus in sociology. The Social Science Journal, 43(1), 55-66. 
-Mintzberg, H. (1991). Mintźberg y la dirección. Madrid. Díaz de Santos.

-Mintzberg, H. (2012). La estructuración de las organizaciones. Barcelona: Ariel.

-Muñoz Miralles, A. (2014). La construcción del carácter frente a las contradicciones del Nuevo Capitalismo en Richard Sennett. Tesis doctoral.

-Piore, M., \& Sabel, Ch. (1984). The Second Industrial Divide: Possibilities for Prosperity. New York: Basics Books.

-Ramis Olivos, A. (2014). Los bienes comunes intangibles en el capitalismo cognitivo. Recerca. Revista de Pensament i Análisi, (15), 109-129.

-Román Maestre, B. (2004). La corrosión del ethos corporativo: reflexiones sobre las condiciones de posibilidad de la ética empresarial. Recerca. Revista de Pensament i Análisi, (4), 137-151.

-Ruiz Olabuénaga, J. I. (1995). Sociología de las organizaciones. Bilbao: Universidad de Deusto.

-Samans, R. \& Davis, N. (2017). Advancing Human-Centred Economic Progress in the Fourth Industrial Revolution. Recuperado de http://www3.weforum.org/docs/WEF_Advancing_Human-Centred_Economic_Progress_WP_2017.pdf

-Sayers, P. (2007). The Concept of Labor: Marx and His Critics. Science and Society, 71(4), 431-454.

-Sennett, R. (1997). The new capitalisme. Social Research, 64(2), 161-180.

-Sennett, R. (1999). Work and social inclusion [conferencia 17 junio de 1999, en el Renner-Institut de Viena].

-Sennett, R. (2000). La corrosión del carácter. Las consecuencias personales del trabajo en el nuevo capitalismo. Barcelona: Anagrama.

-Sennett, R. (2001). La calle y la oficina: dos fuentes de identidad. En A. Giddens y W. Hutton (eds.), En el límite. La vida en el capitalismo global (pp. 247-267). Barcelona: Tusquets.

-Sennett, R. (2002). Cosmopolitanism and the Social Experience of Cities. En S. Vertobec y R. Cohen (eds.), Conceiving Cosmopolitanism. Theory, Context, and Practice (pp. 42-47). Oxford: Oxford University Press.

-Sennett, R. (2003). El Respeto. Sobre la dignidad del hombre en un mundo de desigualdad. Barcelona: Anagrama.

-Sennett, R. (2006a). La cultura del nuevo capitalismo. Barcelona: Anagrama.

-Sennett, R. (2006b). What do you mean by talent? Political Quarterly, 77, 163-167.

-Sennett, R. (2009). El artesano. Barcelona: Anagrama.

-Sennett, R. (2012). Juntos: Rituales, placer y política de la cooperación. Barcelona: Anagrama.

-Smith, N. H. (2007). The hermeneutics of work: on Richard Sennett. Critical Horizons: A Journal of Philosophy and Social Theory, 2(8), 186-204.

-Tallis, R. (2003). The Hand. A philosophical Inquiry into the human being. Edinburgh: Edinburgh University Press.

-Toffler, A. (1995). La empresa flexible. Barcelona: Plaza \& Janes.

-World Economic Forum (2016). The future of jobs: employment, skills and workforce strategy for the fourth industrial revolution. Recuperado de http://www3.weforum.org/docs/WEF_Future_of_Jobs.pdf 
Sumario: 1. Reorientar éticamente las relaciones empresariales en el contexto de las actuales relaciones capitalistas; 2. Los entresijos del Nuevo Capitalismo: la flexibilización del trabajo; 3. La corrosión del carácter en el contexto actual; 4. La artesanía: el despliegue del carácter a través del trabajo; 5 . La artesanía en el marco del Nuevo Capitalismo: orientaciones éticas; 6. Valoraciones de la propuesta de Sennett; Referencias. 